University of Nebraska - Lincoln

DigitalCommons@University of Nebraska - Lincoln

Robert Streubel Papers

Research Papers in Physics and Astronomy

9-28-2015

\title{
Reconfigurable large-area magnetic vortex circulation patterns
}

\author{
Robert Streubel \\ Leibniz-Institut für Festkörper- und Werkstoffforschung Dresden, streubel@unl.edu \\ Florian Kronast \\ Helmholtz-Zentrum Berlin für Materialien und Energie (HZB) \\ Ulrich K. Rößler \\ Leibniz-Institut für Festkörper- und Werkstoffforschung Dresden \\ Oliver G. Schmidt \\ Leibniz-Institut für Festkörper- und Werkstoffforschung Dresden \\ Denys Makarov \\ Leibniz-Institut für Festkörper- und Werkstoffforschung Dresden, d.makarov@ifw-dresden.de
}

Follow this and additional works at: https://digitalcommons.unl.edu/physicsstreubel

Part of the Atomic, Molecular and Optical Physics Commons, Condensed Matter Physics Commons, and the Other Physics Commons

Streubel, Robert; Kronast, Florian; Rößler, Ulrich K.; Schmidt, Oliver G.; and Makarov, Denys, "Reconfigurable large-area magnetic vortex circulation patterns" (2015). Robert Streubel Papers. 17. https://digitalcommons.unl.edu/physicsstreubel/17

This Article is brought to you for free and open access by the Research Papers in Physics and Astronomy at DigitalCommons@University of Nebraska - Lincoln. It has been accepted for inclusion in Robert Streubel Papers by an authorized administrator of DigitalCommons@University of Nebraska - Lincoln. 


\title{
Reconfigurable large-area magnetic vortex circulation patterns
}

\author{
Robert Streubel, ${ }^{1,{ }^{*}}$ Florian Kronast, ${ }^{2}$ Ulrich K. Rößler, ${ }^{3}$ Oliver G. Schmidt, ${ }^{1,4}$ and Denys Makarov ${ }^{1, \dagger}$ \\ ${ }^{1}$ Institute for Integrative Nanosciences, IFW Dresden, 01069 Dresden, Germany \\ ${ }^{2}$ Helmholtz-Zentrum Berlin für Materialien und Energie GmbH, 12489 Berlin, Germany \\ ${ }^{3}$ Institute for Theoretical Solid State Physics, IFW Dresden, 01069 Dresden, Germany \\ ${ }^{4}$ Material Systems for Nanoelectronics, Chemnitz University of Technology, 09107 Chemnitz, Germany \\ (Received 9 December 2014; revised manuscript received 8 September 2015; published 28 September 2015)
}

\begin{abstract}
Magnetic vortices in nanodots own a switchable circulation sense. These nontrivial magnetization configurations can be arranged into extended and interacting patterns. We have experimentally created large arrays of magnetically reconfigurable vortex patterns in nonplanar honeycomb lattices using particle lithography. Optimizing height asymmetry of the vertices and applying an in-plane magnetic field provide means to switch between homocircular and staggered vortex patterns with a potentially high impact on magnonics and spintronics relying on chiral noncollinear spin textures. To this end, exchange coupling of extended vortex lattices with an out-of-plane magnetized layer allows one to realize artificial skyrmionic core textures with controllable circulation and topological properties in extended exchange coupled honeycomb lattices that may pave the way towards magnetic memory and logic devices based on artificial skyrmions.
\end{abstract}

DOI: 10.1103/PhysRevB.92.104431

PACS number(s): 75.70.Kw, 75.75.Cd, 75.75.Fk, 81.16.Dn

\section{INTRODUCTION}

Swirls, vortices, or skyrmions are radial configurations in ordered condensed matter systems. These structures are nontrivial from a topological point of view because of their localized core and noncollinear gross structures. Their circulation sense and eventually their polarity in the core can encode discrete and potentially switchable states [1,2], relevant for applications [3]. Manipulation of individual vortices by means of magnetic $[1,4,5]$ and electric $[6,7]$ fields, other vortices [2,8,9], or magnons [10] has extensively been used to switch between their four possible states. Extended patterns composed of such textures exist in many physical systems. An example is the quasi-long-range order of vortices and antivortices through the Berezinskii-Thouless-Kosterlitz transition in two-dimensional planar spin systems [11,12]. In most of these systems, the extended patterns arise as thermodynamic states. Hence the polarity or circulation sense within the patterns are either dictated by the system itself, as in skyrmion lattices of chiral magnets [13,14], or represent a statistical property of the equilibrium phase. If one could manipulate the configuration of these patterns in extended arrays, it would allow one to control their global properties, e.g., dynamics and interaction with electromagnetic waves or transport properties depending on uniformity or randomness of the local vortexlike configurations in the pattern.

To address these questions, it is necessary to transform such discrete states of nontrivial patterns in an extended magnetic system. Inspired by the shape-driven vortex circulation selection demonstrated in single disks $[15,16]$, we tailor the height asymmetry of vertices in nonplanar extended honeycomb lattices providing means to control the circulation sense of all vortices globally. Hence we can set a homocircular state with all vortices in the extended array displaying the same circulation sense at remanence. In a staggered state,

\footnotetext{
*r.streubel@ifw-dresden.de

†d.makarov@ifw-dresden.de
}

nearest neigbors in the lattice have vortices with different circulation sense. Transitions between these states can be induced by applying appropriate magnetic fields. Capping the vortex patterns with an out-of-plane magnetized film allows one to assemble artificial skyrmionic core textures [17-20] with deterministically switchable circulation and skyrmion number in extended honeycomb lattices.

\section{SAMPLE PREPARATION}

To realize magnetic vortex lattices at a large scale, we apply particle lithography with the unique possibility to induce a three-dimensionality into a deposited film [21] in indentations [22] upon removal of the particle monolayers resembling magnetic cap structures [23-26]. Drop-casting a colloidal solution of spherical polystyrene particles with a diameter of $3 \mu \mathrm{m}$ onto a Si wafer that was exposed to an oxygen plasma leads to an assembly of monolayers with a structural domain size of roughly $100 \times 100 \mu \mathrm{m}^{2}$, which are approximately one thousand unit cells. Adjacent domains possess a slightly rotated lattice orientation. A 100-nm-thick Permalloy (Py, $\mathrm{Ni}_{80} \mathrm{Fe}_{20}$ ) film is sputter-deposited at room temperature (base pressure: $7 \times 10^{-8} \mathrm{mbar}$; Ar pressure: $10^{-3} \mathrm{mbar}$ ) onto the particle array. The propagation vector distribution within the beam ensures deposition both through the interstitials of the monolayers and under the particles. This leads upon removal of the particle monolayer using ultrasonication to a magnetic honeycomb lattice with a periodic thickness modulation as revealed by atomic force microscopy (AFM) measurements [Figs. 1(a) and 1(b)]. The thickest film (35 nm) is located at the vertices and is roughly twice thicker than at the interconnects $(15 \mathrm{~nm})$. Shifting the sample with respect to the center of the beam (along $-60^{\circ}$ ), a height asymmetry is induced in each vertex with largest contributions along $-60^{\circ}$ due to an effectively varying deposition angle distribution [Fig. 1(b)]. The results are obtained for nonplanar honeycomb lattices with a height asymmetry $\mathcal{A}=0.3 \pm 0.1$ if not stated otherwise. The height asymmetry $\mathcal{A}$ is defined as the normalized height difference taken at the left and right edge of the vertex. 

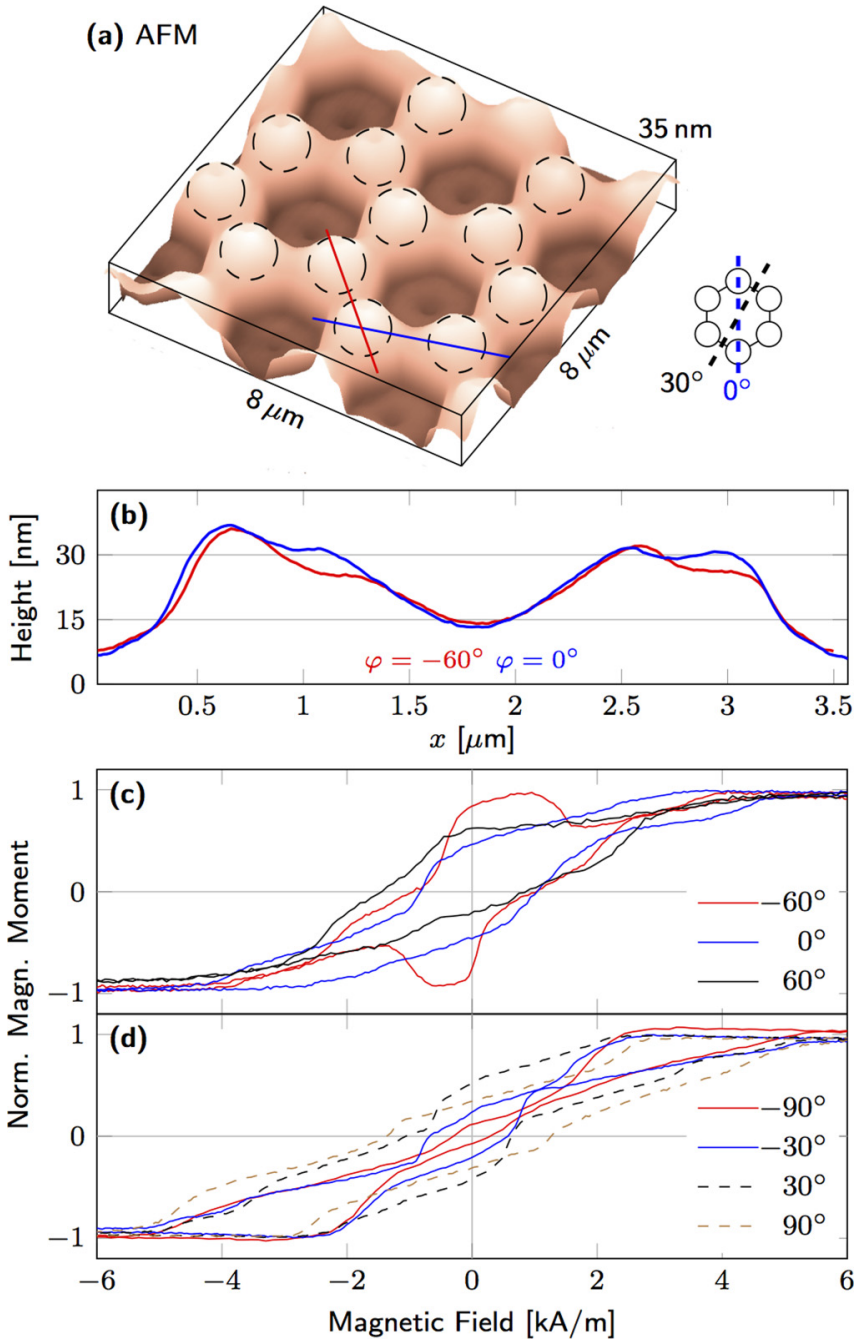

FIG. 1. (Color online) Magnetic nonplanar honeycomb lattice obtained by particle lithography and sputter deposition. (a) Topography of the lattice with a periodicity of $3 \mu \mathrm{m}$ and thicker vertices (dashed circles). (b) Height asymmetry of profiles along various lattice orientations demonstrate effect of off-centered deposition in $-60^{\circ}$ direction. (c), (d) Magnetic hysteresis loops recorded with longitudinal magneto-optical Kerr effect magnetometry at various lattice orientations.

\section{RESULTS AND DISCUSSION}

First, the impact of this geometry on the magnetic properties is investigated with longitudinal magneto-optical Kerr effect (MOKE) magnetometry recording magnetic hysteresis loops with the magnetic field applied in the sample plane at different angles with respect to the magnetic template [Figs. 1(c) and 1(d)]. Figure 1(c) plots the magnetic hysteresis curves acquired along the direction of nearest neighbors. While the curves are of similar shape for $0^{\circ}$ and $60^{\circ}\left(-120^{\circ}\right)$, that one taken along $-60^{\circ}$ possesses distinct features originating from the height asymmetry and mixing in of transverse components. In contrast, hysteresis loops taken at $\pm 30^{\circ}$ and $\pm 90^{\circ}$ resemble those of magnetic vortices in micropatterns. The MOKE magnetometry study suggests that the initial sixfold symmetry of the lattice is superimposed with a uniaxial one along $-60^{\circ}$. Furthermore, the shape of the hysteresis curves taken at, e.g., $30^{\circ}$ indicates that the structural asymmetry allows one to define extended mesoscale honeycomb lattices, where a magnetic vortex is formed in each vertex. Planar structures of this kind have been well investigated experimentally [27-29] and by simulations [30], but do not support chiral spin states. Instead, the in-plane magnetization follows the shape of the antidot array.

\section{A. Stabilization of magnetic vortex states}

The magnetic states in the nonplanar honeycomb lattice are imaged with two complementary techniques offering in-plane or out-of-plane sensitivity, namely x-ray magnetic circular dichroism (XMCD) photoemission electron microscopy (XPEEM) operated at the $L_{3}$ absorption edge of nickel $(852 \mathrm{eV})$ at beamline UE49-PGM-1 (HZB, BESSY II) and frequency-modulated magnetic force microscopy (MFM). The stabilization of the vortex state in the nonplanar honeycomb lattice is proven by recording the magnetization reversal process and monitoring the vortex nucleation with XPEEM [Fig. 2(a)]. The vortex nucleates at the edge of each vertex perpendicular to the field direction after inducing a magnetic domain wall that breaks up into a vortex-antivortex pair [4]. In particular, distinct magnetostatic surface charges, and thus stray fields, at the left and right edge of the vertices with respect to the applied magnetic field due to the existent height asymmetry favor the vortex nucleation at the thicker/steeper edge. In spite of increasing the overall energy by vortex core energy and Zeeman energy, the net energy is significantly lowered due to the reduced stray field energy at the thicker edge. Such a compensation is absent in planar architectures.

Magnified XMCD images in Fig. 2(b) reveal the magnetic patterns of two different states. Corresponding magnetization configurations are illustrated for a dimer (two neighboring vertices with an interconnect). The circulation of two adjacent vortices each located at a vertex may be the same (homocircular) or opposite (staggered). The interpretation of the XMCD contrast is supported by the MFM [Fig. 2(c)]. The recorded MFM images reveal black/white spots in the center of each vertex representing the vortex core polarity. The in-plane magnetization pattern is determined by analyzing both domain wall and magnetic field gradient contrasts [Fig. 2(c)]. The MFM data are summarized in the corresponding schematics, where the locations of vortices (Vs), antivortices (AVs), and half-antivortices at the edges of the interconnects are indicated. Based on statistical analysis of these states no correlation between vortex polarity and circulation is observed. For homocircular configuration, the magnetic contrast at the interconnects between two vertices results in three possible states possessing either antivortex (AV), vortex (V) [Fig. 2(c)], or longitudinal magnetization [Fig. 3(c)]. The staggered state is realized by forming a transverse magnetization in between the vortices, apparent by a domain wall contrast with diamondlike shape, which can be construed as a section of a cross-tie domain wall.

\section{B. Large-area reconfigurable circulation patterns}

These distinct magnetic configurations assemble into circulation patterns distributed throughout the entire structural 
(a) Vortex nucleation and migration

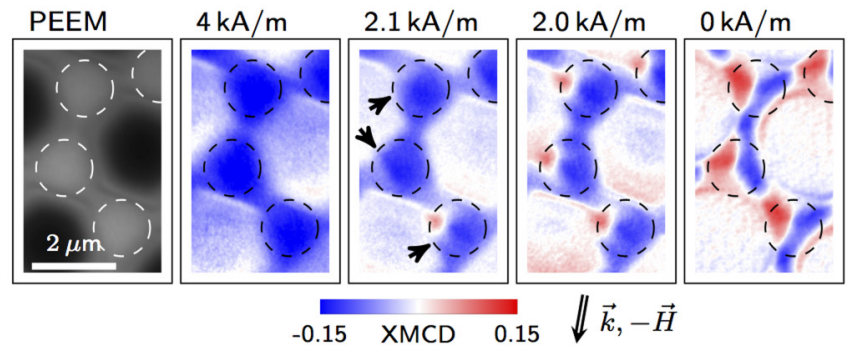

(b) XPEEM contrast

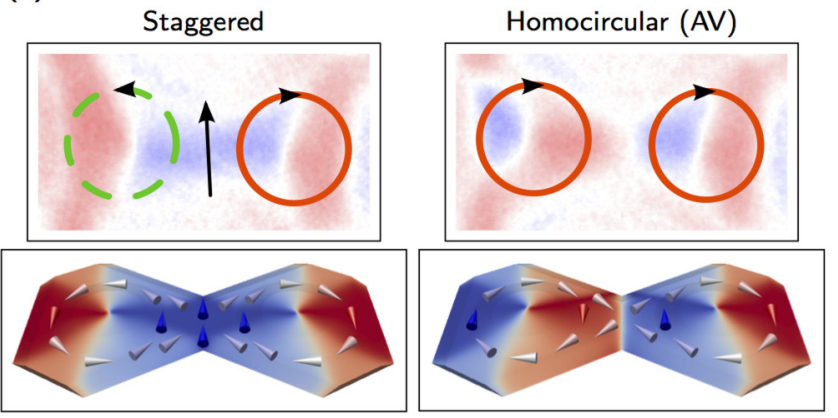

(c) MFM contrast

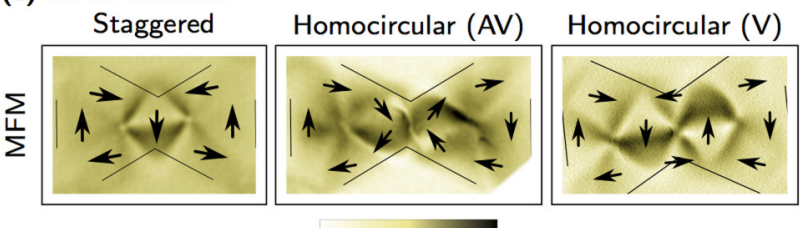

$0 \quad \mathrm{MFM}[\mathrm{Hz}] 4.6$

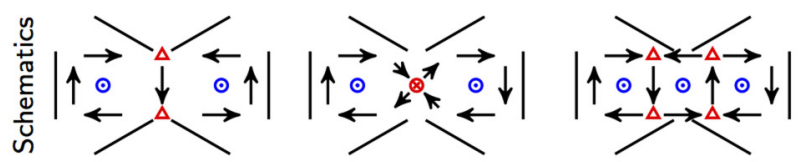

FIG. 2. (Color online) Magnetic nonplanar honeycomb lattice containing a vortex in each vertex. (a) Vortex nucleation in an in-plane magnetic field shown on top of each images recorded with XPEEM at the Ni $L_{3}$ absorption edge. (b) XMCD contrast of staggered and homocircular vortex states visualized in XPEEM. Corresponding magnetization configurations are illustrated for a pair of vertices below. (c) MFM contrast of staggered and homocircular states. In the schematic sketches, vortices are marked by blue circles and antivortices by red circles with cross. Triangles mark half-vortices at the edges.

domain (Fig. 3), which can be reconfigured applying magnetic fields of $4 \mathrm{kA} / \mathrm{m}$ along certain orientations [Fig. 3(a)]. In particular, the staggered vortex configuration is stabilized after ac field demagnetization of the sample [Fig. 3(b)]. This state can be described as a regular lattice of "macrovortices." They consist of circulation patterns around each vertex of the honeycomb lattice formed by the oriented magnetic flux in the domain wall sections that are located at the three interconnects [Fig. 3(b)]. The staggered state can be reconfigured as a homocircular vortex lattice applying the magnetic field along orientations close to $0^{\circ}$. The circulation sense of each vortex in the lattice can be deterministically switched over the whole area by reversing the field direction [compare Figs. 3(c) and 3(f)]. Magnetic fields applied close to $15^{\circ}$ induce defects

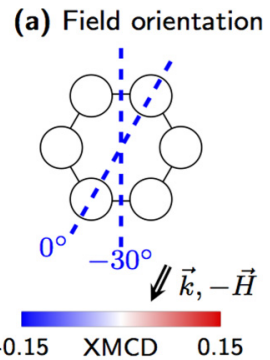

(b) Demagnetized

(c) Remanent: $0^{\circ}$

(d) Remanent: $15^{\circ}$
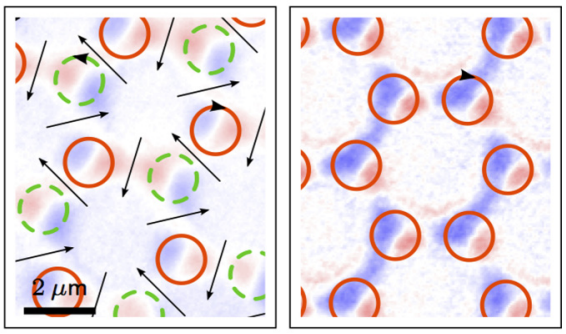

(e) Remanent: $40^{\circ}$

(f) Remanent: $180^{\circ}$
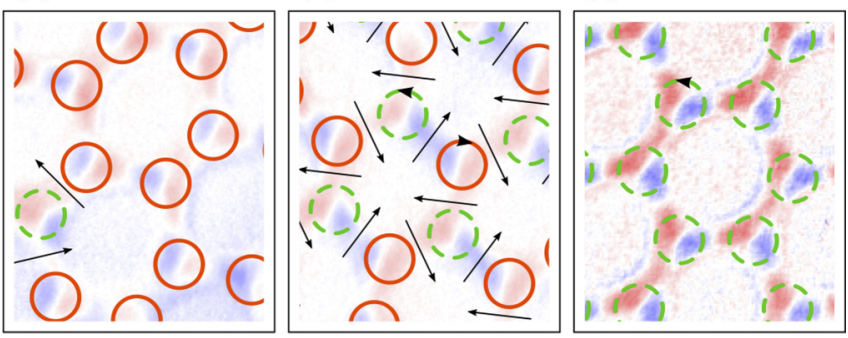

FIG. 3. (Color online) Reconfigurability of vortex circulation patterns throughout the entire structural domain; red-blue shading gives measured XPEEM data. (a) Field direction with respect to the lattice orientation. (b) Staggered pattern obtained by ac demagnetization. (c)-(f) Remanent state after field application $(H=4 \mathrm{kA} / \mathrm{m})$ along different directions, as indicated. The circulation sense of vortices is indicated by circles (red solid for clockwise and green dashed for counterclockwise). Arrows show the orientation of wall sections between pairs of Vs. In the staggered states (b) and (e) the circulation of the magnetic flux forms "macrovortices" around the lattice vertices. For fields $H \| 15^{\circ}$, defects appear in the homocircular pattern, seen by an irregular arrangement of AVs.

in the homocircular vortex circulation pattern [Fig. 3(d)]. Further increase of the angle results in the staggered state [Fig. 3(e)]. Moreover, the magnetization configuration at the interconnects between vertices holds either an antivortex or can become longitudinally magnetized, depending on the field orientation. While applying magnetic fields close to $0^{\circ}$ induces only four antivortices in the interconnects of one hexagon and two longitudinal configurations [Fig. 3(c)], fields at angles up to $15^{\circ}$ generate a regular antivortex lattice on all interconnects [Fig. 3(d)]. Furthermore, a transition from homocircular to randomly distributed circulation patterns without the formation of macrovortex lattices can be stabilized by thermal demagnetization via locally heating above the Curie temperature using femtosecond laser pulses $\left(0.5 \mathrm{~mW} / \mathrm{cm}^{2}\right.$ and 200 fs pulse width) (Fig. 4). The freedom in setting the circulation pattern over large areas is unique to our system. The approach can be extended to generate such circulation patterns also at the submicrometer- and nanoscale by a suitable choice of materials and particle lithography. We demonstrate this by preparing an extended array of magnetic vertices in thickness modulated nominally 40-nm-thick Py films deposited through the particle templates with $500 \mathrm{~nm}$ nonmagnetic spheres [Fig. 5(a)]. The deposition and sample treatment conditions are exactly the same as described in Sec. II. As revealed by the XPEEM imaging [Fig. 5(b)], each vertex in the lattice accommodates a magnetic vortex. 

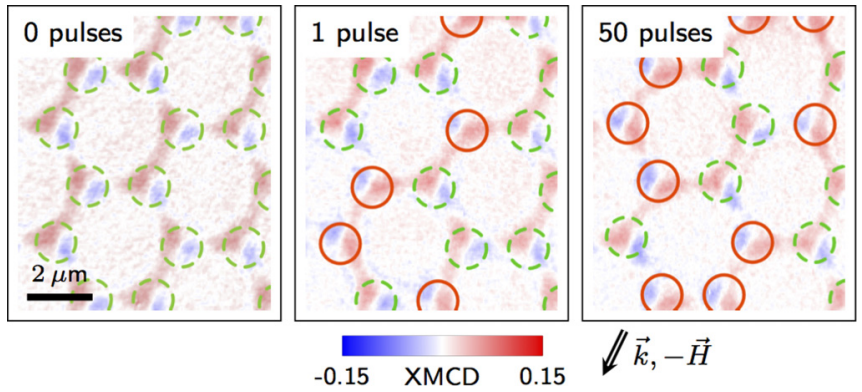

FIG. 4. (Color online) Thermal demagnetization of homocircular patterns by local heating above the Curie temperature using femtosecond laser pulses $\left(0.5 \mathrm{~mW} / \mathrm{cm}^{2}\right.$ and $200 \mathrm{fs}$ pulse width) reveals transition into a disordered vortex circulation pattern. The circulation sense of vortices is indicated by circles (red solid for clockwise and green dashed for counterclockwise).

\section{Role of the height asymmetry}

The reconfigurability relies on the stray field contributions originating from the interplay between the height asymmetry of the vertices and the sixfold symmetry of the hexagonal honeycomb lattice. The latter one prefers a staggered arrangement with a vortex nucleation site located at the edge most normal to the applied magnetic field owing to a minimized energy of the magnetostatic surface charges. The essential requirement of these two competing mechanisms is confirmed by micromagnetic simulations. The magnetic structure within the nonplanar honeycomb lattice is modeled using Nmag v0.2 [31], a FEM/BEM micromagnetic simulator, in combination with the HLib library [32,33]. Two vertices with a thinned interconnect representing the smallest entity (dimer) of the nonplanar honeycomb lattice are modeled (Fig. 6; cross section perpendicular to long dimer axis is shown as insets). The geometry including tilted edges and height asymmetry of the vertices is set similar to the experiment. The thickness of both layers is $25 \mathrm{~nm}$. The periodicity is set to $750 \mathrm{~nm}$, which is equal to an edge length of $l=250 \mathrm{~nm}$. The modeled structures are sufficiently large to generate the same types of magnetic vortex states as in experiment. Statements drawn from these simulations about magnetic states and

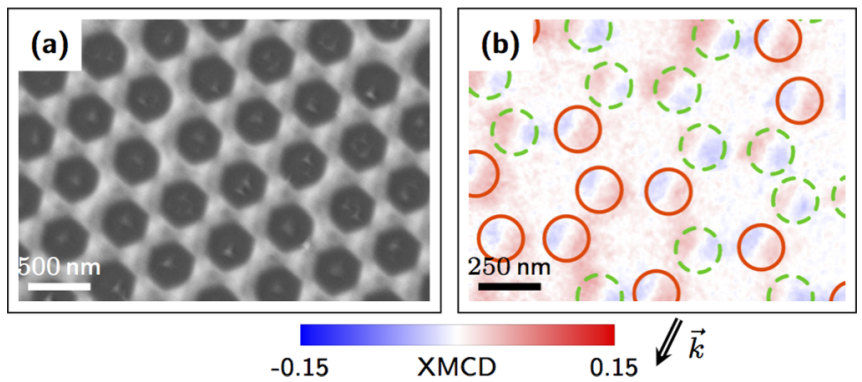

FIG. 5. (Color online) (a) Magnetic nonplanar honeycomb lattice with a period of $500 \mathrm{~nm}$. The polystyrene spheres are partially etched in an oxygen plasma to provide continuity of and thickness gradients in the magnetic film. (b) XMCD signal of Ni recorded by XPEEM reveals a vortex formation in the thicker vertices. The circulation sense of vortices is indicated by circles (red solid for clockwise and green dashed for counterclockwise).
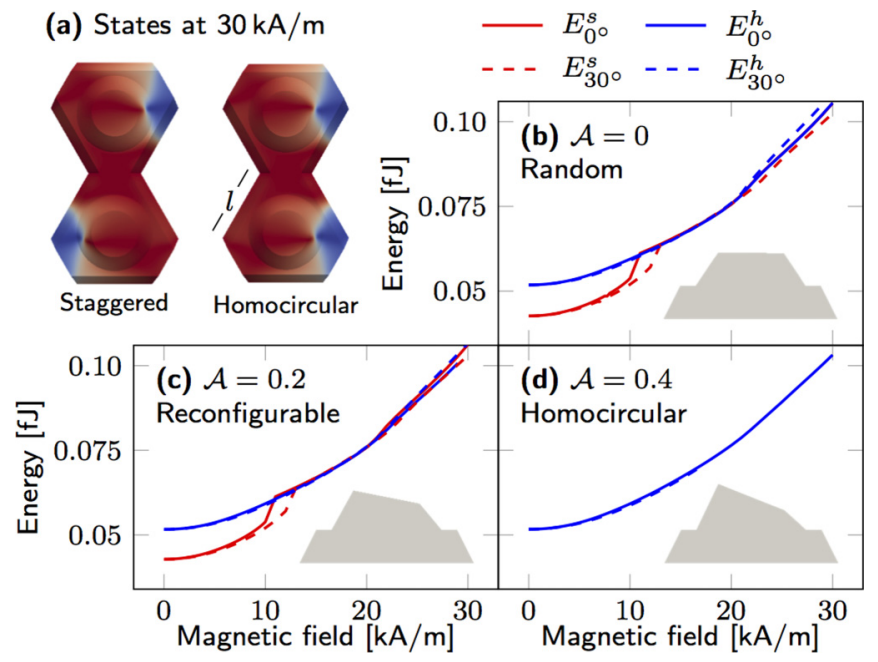

FIG. 6. (Color online) Calculated energies of staggered $\left(E^{s}\right)$ and homocircular $\left(E^{h}\right)$ magnetic states of dimers with edge length $l=$ $250 \mathrm{~nm}$ and varying height asymmetry $\mathcal{A}$ obtained by micromagnetic simulations. (a) Magnetic states with vortices located at the tilted edge when applying an in-plane magnetic field of $30 \mathrm{kA} / \mathrm{m}$. Red and blue shading refer to magnetization aligned parallel and antiparallel with respect to the magnetic field. Panels (b)-(d) plot calculated energies for various height asymmetries with cross sections perpendicular to the long axis of the dimer shown as insets (height $3 \times$ ). (b) $\mathcal{A}=0$ : the staggered state possesses a lower energy at all angles but $0^{\circ}$. (c) $\mathcal{A}=0.2$ : at $0^{\circ}$, energies of homocircular and staggered state cross each other leading to a preference of the homocircular state at large magnetic fields where the vortex core is placed at the tilted edge of the vertex (a). (d) $\mathcal{A}=0.4$ : homocircular pattern is formed at any angle.

energy contributions apply simultaneously to the extended array as the unit cell captures its symmetry. The mesh size is $5 \mathrm{~nm}$. Typical material parameters of Permalloy were used: saturation magnetization $M_{s}=860 \mathrm{kA} / \mathrm{m} ;$ exchange coupling constant $A=13 \mathrm{pJ} / \mathrm{m}$. The energy of the modeled states are linearly integrated over one dimer.

Note that comparing the energies of staggered and homocircular states at remanence is not enough to explain the experimentally and numerically observed reconfigurability due to additional domain wall energy contributions of about $20 \%$ associated to the antivortex/cross-tie formation in the homocircular state (see the Appendix). It is the difference in energy of staggered and homocircular state during vortex nucleation that determines the selection of a circulation pattern and its strong dependence on orientation and height asymmetry. Formation of staggered or homocircular states, even in the simulated models, proceeds through instable nucleation states, when vortices or domain walls are generated. In simulations, we analyze the behavior of these states by comparing their energies under applied magnetic fields, i.e., along distinct branches of the hysteretic evolution of either the staggered or homocircular states (Fig. 6). These simulations identify the stable, metastable, and instable configurations of the magnetic honeycomb lattices with varying height asymmetries. In dimers with a height asymmetry $\mathcal{A}=0.2$, either homocircular or staggered states are observed at remanence when applying in-plane magnetic fields along $0^{\circ}$ and $30^{\circ}$, 
(a) Tailoring height asymmetry of vertices

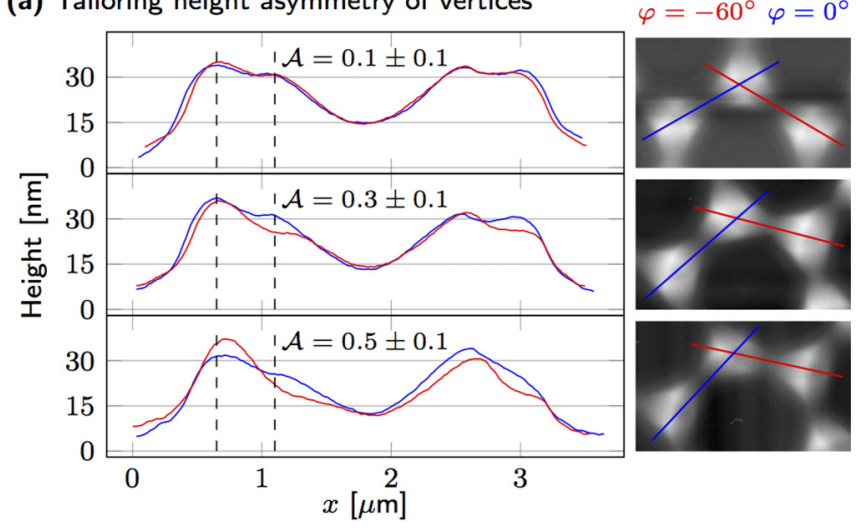

(b) Normalized difference between $\mathrm{cw}$ and $\mathrm{ccw}$ vortex circulation Homocircular Reconfigurable Random $\mathcal{A}=0.5 \pm 0.1 \quad \mathcal{A}=0.3 \pm 0.1 \quad \mathcal{A}=0.1 \pm 0.1$

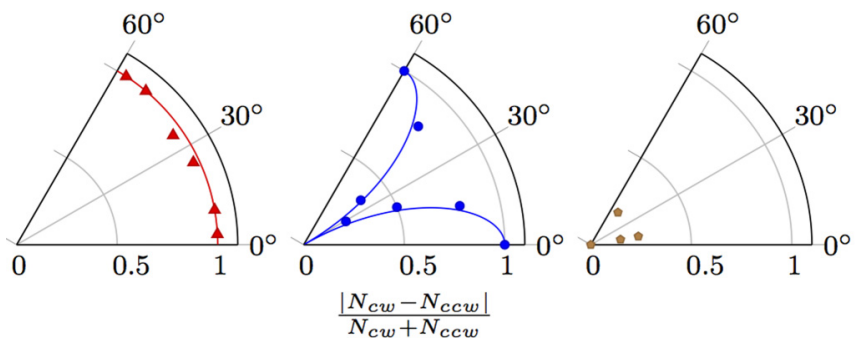

FIG. 7. (Color online) Tailoring vortex circulation selection by experimentally adjusting the height asymmetry of the vertices. (a) Changing the deposition geometry allows for tuning the height asymmetry $\mathcal{A}$ along $-60^{\circ}$. The line profiles are extracted from AFM micrographs shown at the right. (b) Normalized difference between the number of vortices $N_{c w}$ with clockwise (cw) and $N_{c c w}$ counterclockwise (ccw) circulation as a function of height asymmetry and direction of the applied magnetic field. Symbols represent experimental data; connecting curves are guides to the eye.

respectively [Fig. 6(c)]. The corresponding crossing of the energies of staggered and homocircular state shown for a field orientation of $0^{\circ}$ favors the homocircular state at large magnetic fields when the vortices are located at the tilted edges of the vertices. Larger asymmetry values $(\mathcal{A}=0.4)$ stabilize the homocircular state at any lattice orientation with an almost angle-independent energy [Fig. 6(d)]. A vanishing height asymmetry leads to an equal probability to set staggered or homocircular states at $0^{\circ}$ and a staggered state otherwise [Fig. 6(b)].

To provide evidence of these numerical predictions, we experimentally varied the height asymmetry by placing the sphere monolayers during sputter deposition at a location with an offset with respect to the center of the beam along a cross section through the vertex under angle $-60^{\circ}$. The height asymmetry $\mathcal{A}$ is calculated for the experimental data as the normalized height difference along $-60^{\circ}$ taken at the left and right edge of the vertex [dashed vertical lines in Fig. 7(a)]. The line profiles plotted in Fig. 7(a) are extracted from AFM micrographs shown at the right. While deposition close to normal incidence causes a rather symmetric height profile $(\mathcal{A}=0.1 \pm 0.1)$, radially shifted samples reveal an enlarged height asymmetry along $-60^{\circ}$ up to $\mathcal{A}=0.5 \pm 0.1$ [Fig. 7(a)]. Samples with $\mathcal{A} \approx 0.5$ reveal independently of the field direction a homocircular state [Fig. 7(b)]. Applying in-plane magnetic fields to samples with a moderate height asymmetry $\mathcal{A}=0.3 \pm 0.1$ (Fig. 3) nucleates homocircular vortex circulation patterns at $0^{\circ}$ and $180^{\circ}$, and staggered states at angles close to $30^{\circ}$ [Fig. 7(b)]. On the other hand, samples with an almost vanishing height asymmetry $(\mathcal{A}=0.1 \pm 0.1)$ possess randomly distributed circulation patterns with an equal number of left- and right-handed vortex circulations [Fig. 7(b)].

\section{Imprinting noncollinear spin textures}

In the following, we demonstrate that asymmetric Py honeycomb lattices $(\mathcal{A}=0.3 \pm 0.1)$ stabilize via imprint noncollinear spin textures with controllable circulation patterns in extended out-of-plane magnetized thin films. This way, vortices and donut states resembling skyrmionic core textures with distinct topological properties [19,20] are formed. Rotation symmetry and nonvanishing normal magnetization components combined with a larger core size of the imprinted states allow one to simultaneously access circulation and polarity of the Py vortex lattices due to interlayer exchange coupling. The hard-magnetic out-of-plane magnetized multilayer stack $\left([\mathrm{Co}(0.4 \mathrm{~nm}) / \mathrm{Pd}(0.7 \mathrm{~nm})]_{5}\right)$ providing full remanence after saturating along its easy axis is sputter-deposited (base pressure: $7 \times 10^{-8}$ mbar; Ar pressure: $10^{-3}$ mbar, rate: $1 \AA / \mathrm{s}$ ) directly onto the honeycomb lattice after Py deposition and removal of the monolayers [Fig. 8(a)]. Contrary to particle lithography, a modulation of thickness and magnetic properties of the $\mathrm{Co} / \mathrm{Pd}$ layers does not occur as the honeycomb surface is tilted by $3^{\circ}$ at most with respect to vertical orientation. Previous studies on curved magnetic cap structures demonstrated a preservation of magnetic properties for surface angles up to $50^{\circ}[23,24]$.

The magnetic remanent states after saturation in an out-ofplane magnetic field of $40 \mathrm{kA} / \mathrm{m}$ are visualized with XPEEM at the $\mathrm{Co} L_{3}$ absorption edge [Fig. 8(b)]. The interlayer exchange coupling via a 2-nm-thick Pd spacer separating $\mathrm{Py}$ and $\mathrm{Co} / \mathrm{Pd}$ subsystems imprints vortices into the $\mathrm{Co} / \mathrm{Pd}$ system, which are reflected by the typical dipolar XMCD contrast [Fig. 8(b1)] [25,26]. The circulation of the $\mathrm{Co} / \mathrm{Pd}$ vortices arranges in patterns with staggered symmetry as in the underlying Py template. The weak XMCD contrast originating from secondary electrons emanating from the Py film buried by a 10-nm-thick capping layer allowed for a clear identification of 10 out of 35 Py vortices located in the field of view of the microscope $(15 \mu \mathrm{m})$ all possessing the same circulation as the overlaying $\mathrm{Co} / \mathrm{Pd}$ spin system. However, the majority of vertices exhibits more complex XMCD contrast patterns with a prominent fine structure in the region separating blue and red contrast [Figs. 8(b2) and 8(b3)].

To shed light onto these patterns, we calculate the XMCD contrast for shallow X-ray incidence angles characteristic for our XPEEM device ( $74^{\circ}$ with respect to surface normal) of planar vortex and donut textures shown in Fig. 8(c). The corresponding magnetic states in disks with a diameter of $650 \mathrm{~nm}$ are obtained from micromagnetic simulations using an interlayer exchange interaction of 1 and $0.4 \mathrm{~mJ} / \mathrm{m}^{2}$ for vortex and donut states, respectively, and materials parameters described in Ref. [19,20]. This calculation reveals the role of 
(a) $[\mathrm{Co} / \mathrm{Pd}] / \mathrm{Pd} / \mathrm{Py}$ heterostructure $(\mathcal{A}=0.3 \pm 0.1)$

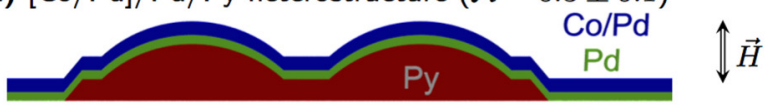

(b) Experimental XMCD contrast of remanent states
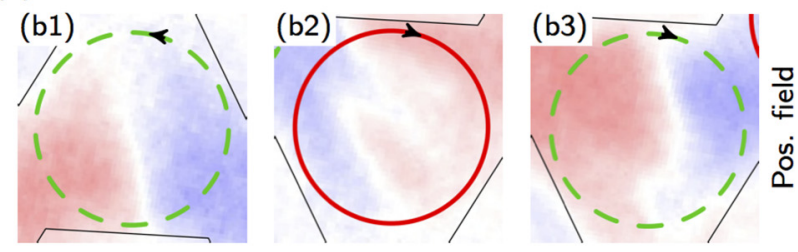

Vortex $\leftrightarrow$ vortex

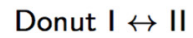

Donut I $\leftrightarrow$ I
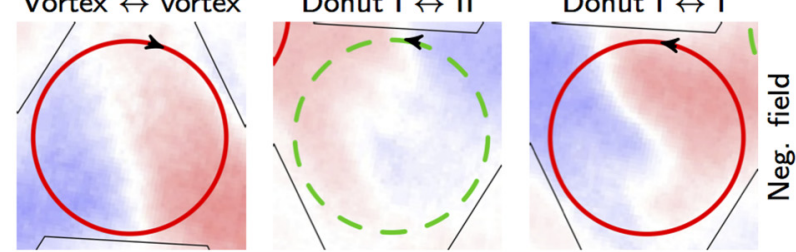

(c) Calculated XMCD contrast
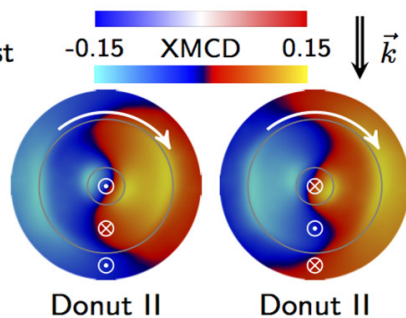

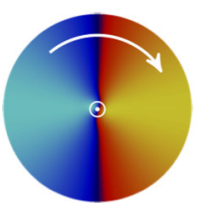

Vortex

$p=1$
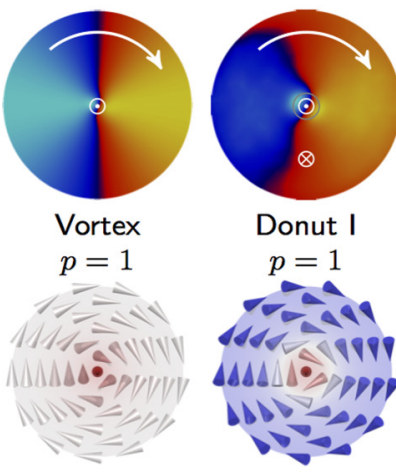

Donut I
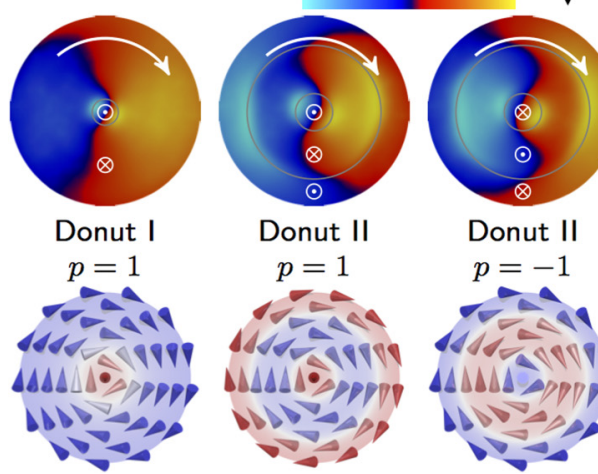

Donut II

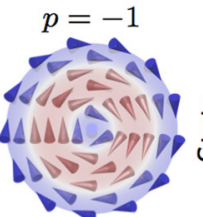

FIG. 8. (Color online) Imprinting noncollinear spin textures. (a) Cross section through honeycomb lattice after capping with $\mathrm{Co} / \mathrm{Pd}$ multilayer stack. (b) Remanent states in the Co/Pd layers visualized with XPEEM after applying magnetic fields $(H= \pm 40$ $\mathrm{kA} / \mathrm{m}$ ) normal to the sample surface. The circulation sense of vortices is indicated by circles (red solid for clockwise and green dashed for counterclockwise). (c) Correlation with the calculated XMCD contrast of the shown magnetic states reveals circulation and polarity switching. Normal magnetization components pointing up and down are indicated by circles and crosses, or shaded in red and blue color, respectively.

locally varying normal magnetization components (indicated by circles and crosses) to distinct XMCD contrast features [Fig. 8(c)]. Accordingly, we identify the imprinted magnetic states [Figs. 8(b2) and 8(b3)] as donut states type I and II, which possess distinct topological properties reflected by their skyrmion number [19]. In agreement with micromagnetic simulations [19], donut state I and II can be transformed into each other by applying out-of-plane magnetic fields of $\pm 40 \mathrm{kA} / \mathrm{m}$ [Fig. 8(b2)]. The circulation of imprinted vortex and donut states is deterministically set according to the field direction owing to the exchange coupling to the underlaying Py vortex. In addition, switching of donut state I from one circulation sense and polarity to its opposite form is observed [Fig. 8(b3)]. This transformation is only possible if the vortex is expelled from the Py vertex during the magnetization reversal of the $\mathrm{Co} / \mathrm{Pd}$ stack, which might be caused by local in-plane field components appearing due to the three-dimensionality of the nonplanar honeycomb lattice and small vortex annihilation fields $[4 \mathrm{kA} / \mathrm{m}$, Fig. 1(d)]. The statistically ( $\approx 50 \%)$ observed polarity switching confirms this statement.

The observation of skyrmionic core textures located at each vertex whose circulation can be reconfigured by an external magnetic field paves the way towards artificial skyrmion lattices based on the present hybrid system. The in-plane circulation of the imprinted chiral texture for each skyrmionic core in the lattice is set according to the vortex circulation, while the out-of-plane magnetization is determined by the magnetic field applied normal to the sample surface. For the chosen parameters of the films in the stack, the $\mathrm{Co} / \mathrm{Pd}$ multilayers are split into a multidomain state at remanence. Adjusting the direction and strength of an external magnetic field provides means to switch either partially (donut state II) or entirely (donut state I) the normal $\mathrm{Co} / \mathrm{Pd}$ magnetization except for the core region. If interlayer exchange coupling is properly adjusted, the corresponding magnetic domain pattern resembles donut states at each vertex.

In conclusion, we have generated magnetic vortex lattices on a large scale possessing deterministically controllable vortex circulation patterns, such as homocircular and staggered state. In particular, magnetic dynamics and transport properties for both magnons and electric charges may be manipulated by choosing either state. Structures grown on top of arbitrary films allow one to realize heterostructures where the magnetic vortex patterns can be used to manipulate other components in the stack. The stabilization of skyrmionic core textures with controllable topological properties in extended honeycomb lattices is appealing for field- or current-driven displacements of noncollinear textures, which is an essential requirement to realize memories or logic devices based on artificial skyrmionic textures. On superconducting films, the pattern may locally exert a magnetic proximity effect or influence the flux-line lattice by the localized dipolar stray field of the vortices.

\section{ACKNOWLEDGMENTS}

We thank C. F. Reiche (IFW Dresden) for support with MFM, and Dr. T. Mühl (IFW Dresden) and Dr. V. P. Kravchuk (BITP Kiev) for fruitful discussions on the MFM contrast and on the analytical treatment, respectively. This work is financed via the European Research Council under European Union's Seventh Framework program (FP7/2007-2013)/ERC Grant agreement No. 306277 and the Future and Emerging Technologies (FET) programme under FET-Open Grant No. 618083. We thank HZB for the allocation of synchrotron radiation beamtime.

\section{APPENDIX: ANALYTICAL APPROACH TO ASSESS ENERGIES OF STAGGERED AND HOMOCIRCULAR STATES}

The energies of the staggered and homocircular (AV) state are roughly approximated by taking into account contributions of magnetic vortices and magnetic domain walls. Typical material parameters for Permalloy are used: $A=13 \mathrm{pJ} / \mathrm{m}$, $M_{s}=860 \mathrm{kA} / \mathrm{m}$, and $l_{e x} \approx 10 \mathrm{~nm}$. 
The wall energy of a 1D magnetic $90^{\circ}$ Nèel wall with a length $L$ and a mean thickness $\bar{h}$ reads [34]

$$
E_{d w} \approx 12 \% \times 4 \bar{h} L \sqrt{\frac{1}{2} A \mu_{0} M_{s}^{2}},
$$

and becomes for the staggered configuration $E_{d w}=0.1 \mathrm{fJ}$ with $\bar{h}=25 \mathrm{~nm}$ and $L=2 \times 2 \mu \mathrm{m}$. In addition to this contribution describing the diamond state, further $90^{\circ}$ domain walls belonging to the cross-tie/antivortex formation have to be considered for homocircular states. With $L=(2.0+0.5) \mu \mathrm{m}$ and $\bar{h}=25 \mathrm{~nm}$, it becomes $E_{\text {cross }}=0.06 \mathrm{fJ}$.

The exchange energy of a vortex/antivortex $(q=1)$ constitutes of core and tail contributions with the radius $R$ :

$$
E_{v, a v}=2 \pi A \bar{h}\left(1.76+\ln \frac{R}{l}\right) .
$$

The energy of the localized vortex at the vertex is $E_{v}=13.9 \mathrm{aJ}$ using $\bar{h}=30 \mathrm{~nm}$ and $R_{v}=500 \mathrm{~nm}$. For the antivortex located in between the vertices, an energy of $E_{a v}=5.8 \mathrm{aJ}$ is obtained using $\bar{h}=15 \mathrm{~nm}$ and $R_{a v}=200 \mathrm{~nm}$. The smaller expansion of the antivortex tail is due to the geometry of the honeycomb lattice. The energy contribution of an antivortex located outside the pattern is $E_{a v}^{q=\frac{1}{2}}=1.8 \mathrm{aJ}$.

The overall energy of the staggered and homocircular (AV) state with a periodicity of $3 \mu \mathrm{m}$ is

$$
\begin{aligned}
& E^{s}=2 E_{v}+E_{d w}+2 E_{a v}^{q=\frac{1}{2}}=0.13 \mathrm{fJ}, \\
& E^{h}=2 E_{v}+E_{d w}+E_{\text {cross }}+E_{a v}=0.19 \mathrm{fJ},
\end{aligned}
$$

which is equal to an energy difference of $\Delta E=0.06 \mathrm{fJ} \sim$ $37 \%$. For a dimer with a periodicity of $750 \mathrm{~nm}$, as considered in micromagnetic simulations, the energies are

$$
\begin{aligned}
E^{s} & =0.05 \mathrm{fJ}, \\
E^{h} & =0.06 \mathrm{fJ}, \\
\Delta E & =0.01 \mathrm{fJ} \approx 20 \% .
\end{aligned}
$$

[1] B. Pigeau, G. de Loubens, O. Klein, A. Riegler, F. Lochner, G. Schmidt, and L. W. Molenkamp, Nat. Phys. 7, 26 (2011).

[2] H. Jung, Y.-S. Choi, K.-S. Lee, D.-S. Han, Y.-S. Yu, M.-Y. Im, P. Fischer, and S.-K. Kim, ACS Nano 6, 3712 (2012).

[3] J.-G. Zhu, Y. Zheng, and G. A. Prinz, J. Appl. Phys. 87, 6668 (2000).

[4] B. Van Waeyenberge, A. Puzic, H. Stoll, K. W. Chou, T. Tyliszczak, M. Fähnle, H. Brückl, K. Rott, G. Reiss, I. Neudecker, D. Weiss, C. H. Back, and G. Schütz, Nature (London) 444, 461 (2006).

[5] K.-S. Lee, S.-K. Kim, Y.-S. Yu, Y.-S. Choi, K. Y. Guslienko, H. Jung, and P. Fischer, Phys. Rev. Lett. 101, 267206 (2008).

[6] K. Yamada, S. Kasai, Y. Nakatani, K. Kobayashi, H. Kohno, A. Thiaville, and T. Ono, Nat. Mater. 6, 269 (2007).

[7] T. Kamionka, M. Martens, K. W. Chou, M. Curcic, A. Drews, G. Schütz, T. Tyliszczak, H. Stoll, B. Van Waeyenberge, and G. Meier, Phys. Rev. Lett. 105, 137204 (2010).

[8] S. Sugimoto, Y. Fukuma, S. Kasai, T. Kimura, A. Barman, and Y. C. Otani, Phys. Rev. Lett. 106, 197203 (2011).

[9] A. Vogel, T. Kamionka, M. Martens, A. Drews, K. W. Chou, T. Tyliszczak, H. Stoll, B. Van Waeyenberge, and G. Meier, Phys. Rev. Lett. 106, 137201 (2011).

[10] H. G. Bauer, M. Sproll, C. H. Back, and G. Woltersdorf, Phys. Rev. Lett. 112, 077201 (2014).

[11] J. M. Kosterlitz and D. J. Thouless, J. Phys. C: Solid State Phys. 6, 1181 (1973).

[12] Z. Hadzibabic, P. Krüger, M. Cheneau, B. Battelier, and J. Dalibard, Nature (London) 441, 1118 (2006).

[13] A. N. Bogdanov and D. A. Yablonskii, Sov. Phys. JETP 68, 101 (1989).

[14] A. N. Bogdanov and U. K. Rößler, Phys. Rev. Lett. 87, 037203 (2001).
[15] S. Yakata, M. Miyata, S. Nonoguchi, H. Wada, and T. Kimura, Appl. Phys. Lett. 97, 222503 (2010).

[16] V. Uhlir, M. Urbanek, L. Hladik, J. Spousta, M.-Y. Im, P. Fischer, N. Eibagi, J. J. Kan, E. E. Fullerton, and T. Sikola, Nat. Nanotechnol. 8, 341 (2013).

[17] L. Sun, R. X. Cao, B. F. Miao, Z. Feng, B. You, D. Wu, W. Zhang, A. Hu, and H. F. Ding, Phys. Rev. Lett. 110, 167201 (2013).

[18] J. Li, A. Tan, K. Moon, A. Doran, M. Marcus, A. Young, E. Arenholz, S. Ma, R. Yang, C. Hwang, and Z. Qiu, Nat. Commun. 5, 4704 (2014).

[19] R. Streubel, L. Han, M.-Y. Im, F. Kronast, U. K. Rößler, F. Radu, R. Abrudan, G. Lin, O. G. Schmidt, P. Fischer, and D. Makarov, Sci. Rep. 5, 8787 (2015).

[20] R. Streubel, P. Fischer, M. Kopte, O. G. Schmidt, and D. Makarov, Appl. Phys. Lett. 107, 112406 (2015).

[21] A. Kosiorek, W. Kandulski, P. Chudzinski, K. Kempa, and M. Giersig, Nano Lett. 4, 1359 (2004).

[22] D. Makarov, L. Baraban, I. L. Guhr, J. Boneberg, H. Schift, J. Gobrecht, G. Schatz, P. Leiderer, and M. Albrecht, Appl. Phys. Lett. 90, 093117 (2007).

[23] M. Albrecht, G. Hu, I. L. Guhr, T. C. Ulbrich, J. Boneberg, P. Leiderer, and G. Schatz, Nat. Mater. 4, 203 (2005).

[24] T. C. Ulbrich, D. Makarov, G. Hu, I. L. Guhr, D. Suess, T. Schrefl, and M. Albrecht, Phys. Rev. Lett. 96, 077202 (2006).

[25] R. Streubel, D. Makarov, F. Kronast, V. Kravchuk, M. Albrecht, and O. G. Schmidt, Phys. Rev. B 85, 174429 (2012).

[26] R. Streubel, V. P. Kravchuk, D. D. Sheka, D. Makarov, F. Kronast, O. G. Schmidt, and Y. Gaididei, Appl. Phys. Lett. 101, 132419 (2012).

[27] E. T. Papaioannou, V. Kapaklis, E. Melander, B. Hjörvarsson, S. D. Pappas, P. Patoka, M. Giersig, P. Fumagalli, A. GarciaMartin, and G. Ctistis, Opt. Express 19, 23867 (2011). 
[28] J. Kim, S.-G. Cho, Y.-h. Choa, G. J. Shin, and J. Kim, Thin Solid Films 520, 3013 (2012).

[29] A. S. Hall, S. A. Friesen, and T. E. Mallouk, Nano Lett. 13, 2623 (2013).

[30] S. V. Kostinski, E. R. Chen, and M. P. Brenner, Phys. Rev. Lett. 112, 235502 (2014).

[31] T. Fischbacher, F. Matteo, G. Bordignon, and H. Fangohr, IEEE Trans. Magn. 43, 2896 (2007).
[32] W. Hackbusch, Hierarchische Matrizen: Algorithmen und Analysis (Springer, New York, 2009).

[33] S. Börm, Efficient Numerical Methods for Non-local Operators: $H^{2}$-Matrix Compression, Algorithms and Analysis, EMS Tracts in Mathematics Vol. 14 (European Mathematical Society, Zurich, 2010).

[34] A. Hubert and R. Schäfer, Magnetic Domains (Springer, New York, 1998). 